\title{
Three-dimensional Numerical Simulations of Straight-Bladed Vertical Axis Tidal Turbines Investigating Power Output, Torque Ripple and Mounting Forces
}

Philip Marsh ${ }^{1 a}$, Dev Ranmuthugala ${ }^{2}$, Irene Penesis ${ }^{1}$ and Giles Thomas ${ }^{3}$

${ }^{1}$ National Centre for Maritime Engineering and Hydrodynamics, Australian Maritime College, University of Tasmania. Locked Bag 1399, Launceston Tasmania 7250, Australia

${ }^{2}$ National Centre for Ports and Shipping, Australian Maritime College, University of Tasmania. Locked Bag 1399, Launceston Tasmania 7250, Australia

${ }^{3}$ UCL Mechanical Engineering, University College London, Torrington Place, London WC1E 7JE

a Corresponding author: marshp@amc.edu.au. Locked Bag 1395, Launceston Tasmania 7250, Australia. Phone: (+61) 401639059

\begin{abstract}
Three straight-bladed vertical axis turbine designs were simulated using ThreeDimensional (3D) transient Computational Fluid Dynamics (CFD) models, using a commercial Unsteady Reynolds Averaged Navier-Stokes (URANS). The turbine designs differed in support strut section, blade-strut joint design and strut location to evaluate their effect on power output, torque fluctuation levels and mounting forces. Simulations of power output were performed and validated against Experimental Fluid Dynamics (EFD), with results capturing the impacts of geometrical changes on turbine power output. Strut section and blade-strut joint design were determined to significantly influence total power output between the three turbine designs, with strut location having a smaller but still significant effect. Maximum torque fluctuations were found to occur around the rotation speed corresponding to maximum power output and fluctuation levels increased with overall turbine efficiency. Turbine mounting forces were also simulated and successfully validated against EFD results. Mounting forces aligned with the inflow increased with rotational rates, but plateaued due to reductions in shaft drag caused by rotation and blockage effects. Mounting forces perpendicular to the inflow were found to be $75 \%$ less than forces aligned with the inflow. High loading force fluctuations were found, with maximum values $40 \%$ greater than average forces.
\end{abstract}


Keywords: Vertical Axis Turbine, Computational Fluid Dynamics, Three-Dimensional, Tidal Power, Ocean Power, Power Output.

\section{Highlights}

- Three straight-bladed turbine designs simulated using Computational Fluid Dynamics

- Strut section, blade-strut joint design and strut location significantly impacted power output

- Large torque fluctuations occurred around the rotation speed of maximum power

- Turbine mounting forces were found to vary with turbine efficiency

\section{Introduction}

A combination of social, environmental and economic interests is driving research into renewable energy, the production of which is a central facet of sustainable human development. The energy held within the ocean could be harnessed in a renewable, sustainable and economic manner, with over 50 devices proposed to extract this energy [1]. However, of these devices only a few have been constructed or are near commercial feasibility [2]. Vertical and horizontal axis turbines are among these devices being developed to transform the ocean's kinetic energy contained within tides and currents into usable energy forms [2].

Vertical axis turbines as shown in Figure 1 have two key advantages when compared to horizontal axis designs: they are flow-directional independent [3]; and all electrical components can be installed above the free surface of the water, thus simplifying installation and maintenance [4]. With increased interest in vertical axis turbines, driven in part by the plateauing of efficiency of horizontal axis designs [5], further research into the development of improved turbine designs is required. This has proven challenging due to the complexity of vertical axis turbine hydrodynamic flow fields which are notoriously difficult to predict [5]. 


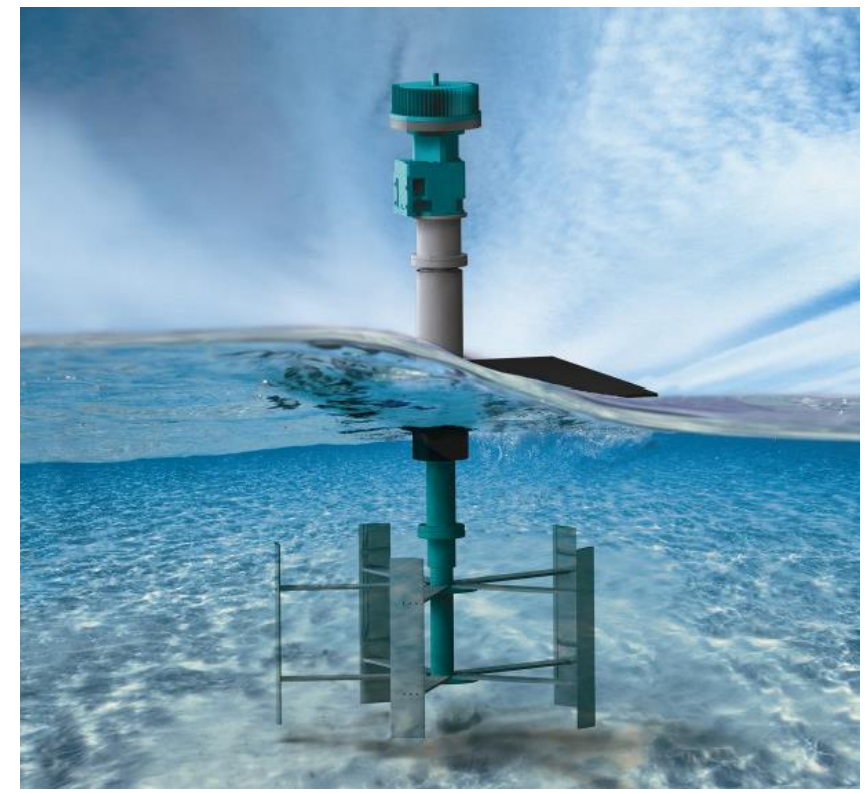

Figure 1: Vertical Axis turbine for tidal and current power generation [6]

The complexity of vertical axis turbine hydrodynamics is due to three distinct phenomena that generate high levels of unsteady flow and thus complex vortex shedding and flow diffusion effects:

- continually varying blade angles of attack;

- blade-wake interactions; and

- $\quad$ strut effects.

At low rotational rates the flow is dominated by high levels of static and dynamic stall as the blade angles of attack widely vary due to the low blade velocities relative to the inflow velocity [7]. At higher rotational rates the flow field is dominated by wake interaction as the blades traverse through the disturbed wake and shed vortices of previous blades [5]. Additionally, as rotational rates increase the influence of strut drag increases, reducing the overall torque generated [8]. These complex flow field phenomena and the resultant vortex shedding and flow diffusion effects must be accurately captured by the simulation models used in order to accurately simulate turbine hydrodynamics and thus turbine efficiency.

A wide variety of numerical models can be used to simulate vertical axis turbine performance and hydrodynamics, ranging from reduced order blade-element based models [7-9], vortex methods [10, 11], two-dimensional (2D) Computational Fluid Dynamics (CFD) models [12], quasi 2D or 2.5D Large Eddy Scale (LES) simulations which treat the blades as possessing infinite length [13], and 
three-dimensional (3D) CFD models [14,15], with increasing levels of simulation complexity and computational resource requirements. Commercial CFD software such as ANSYS Fluent and CFX are commonly used to simulate turbine power output and hydrodynamics [3,8,12-18], with most CFD simulations performed in 2D as 3D models require lengthy simulation times [10,13,17]. However, 2D (and by extension 2.5D) CFD simulations often unsatisfactorily estimate power output, as the losses due to strut drag and finite blade lengths are not simulated $[8,15]$. To accurately capture all hydrodynamic phenomena, CFD models should include all significant geometrical features, necessitating the use of full 3D simulation approaches. Although empirical corrections for 2D CFD and blade-element models are available $[8,10,16]$ in this work evaluations were conducted to determine whether recent advances in distributed computing now make 3D CFD simulation approaches feasible.

In this work simulations were performed using 3D CFD models to predict the power output, torque fluctuations and loading characteristics of three straight-bladed vertical axis turbines. These designs used the same blade section but different strut sections, blade-strut joint designs and strut locations to evaluate the effect of geometrical changes on turbine performance. Power output curves were generated for all turbines, two of which were compared to Experimental Fluid Dynamics (EFD) results to validate simulation models. The levels of torque fluctuations and total mounting forces were also characterised to determine their relationship with strut location, strut section and total power output. All CFD methods were verified using mesh independence criteria with results validated against EFD where possible.

\section{Numerical Simulations}

Transient time-accurate 3D CFD simulations were conducted in ANSYS CFX software utilising the URANS based Shear Stress Transport (SST) turbulence model using an element-based finite volume approach [19]. Ansys CFX was utilised as it is commonly used in industry and its formulation allows for efficient distribution on computing clusters to reduce total simulation time [19].Unsteady models were used due to the high levels of unsteady flow caused by the rotation of the turbine, with the fluid modelled as an incompressible fluid as all flow velocities were significantly less than Mach 0.3.

\subsection{Turbine Geometry}


Three straight-bladed vertical axis turbine designs were simulated, labelled turbines A, B and C, with the overall geometrical dimensions and configurations as outlined in Table 1, which were based on the EFD turbines of Rawlings [20]. These turbines were simulated as they allowed for comparisons of power output for varying geometrical designs as well as validation of simulation results against EFD. All turbines had two struts per blade, with strut section, location and blade-strut joint design details outlined in Figure 2.

Table 1: Common geometrical features of the three turbine configurations [20]

\begin{tabular}{cc}
\hline Geometry & Dimensions \\
\hline Number of blades & 3 \\
Number of struts & 2 per blade \\
Blade section & NACA $63_{4} 021$ \\
Blade chord & $0.065 \mathrm{~m}$ \\
Radius & $0.457 \mathrm{~m}$ \\
Blade span & $0.686 \mathrm{~m}$
\end{tabular}

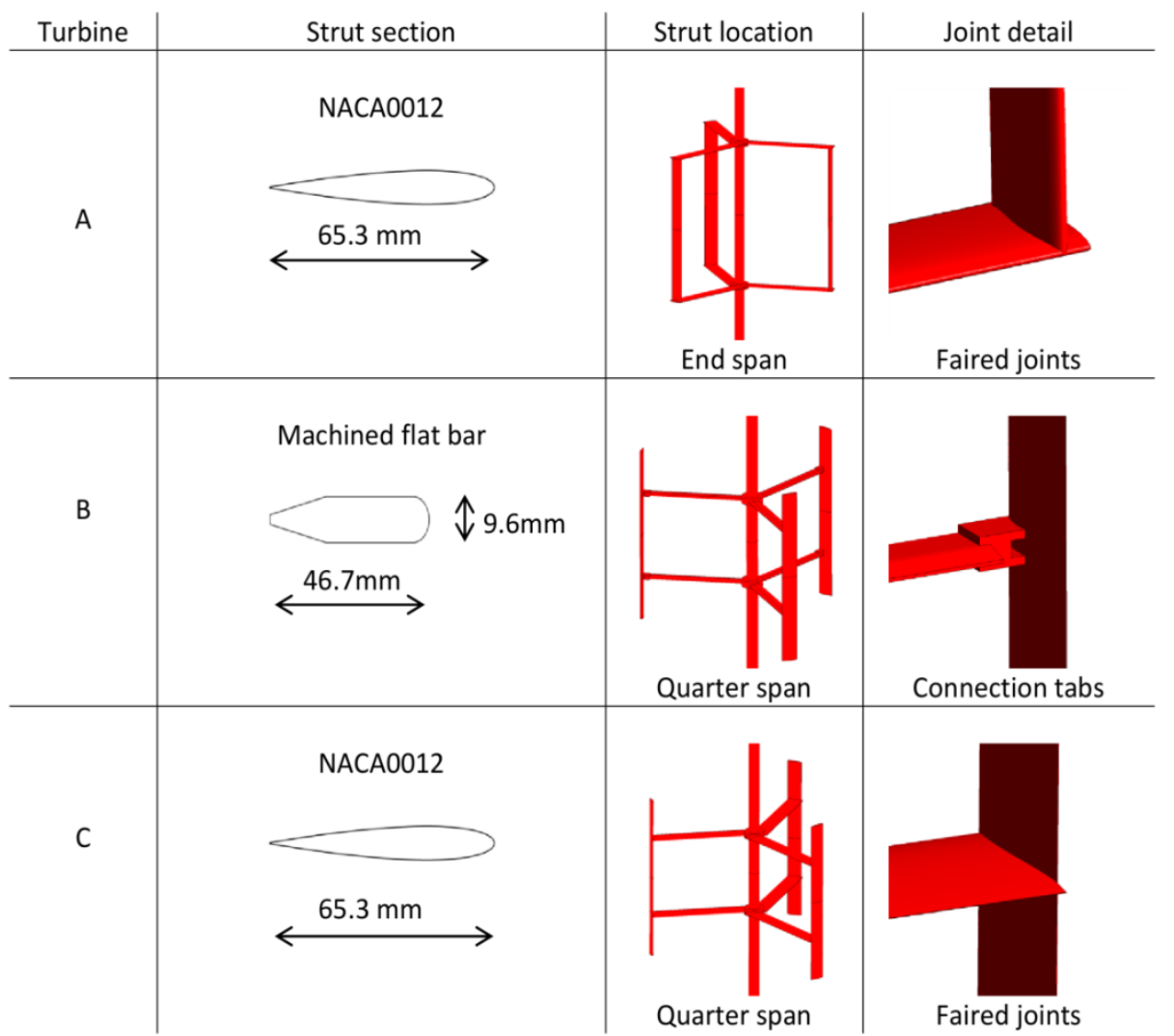

Figure 2: Turbines A, B, and C strut section, strut location and blade-strut joint detail [20] 


\subsection{Key Performance Parameters}

A number of performance parameters were investigated to enable the quantification of turbine efficiency and operating loading characteristics. Turbine power output was expressed as a nondimensionalised power coefficient $C_{p}$, where,

$$
C_{p}=\lambda C_{m}
$$

where tip speed ratio $\lambda$ was defined as,

$$
\lambda=r \omega / V
$$

where $\omega$ was the turbine rotational rate, $r$ was the turbine radius, and $V$ was the inflow velocity. The turbine torque coefficient $C_{m}$ was determined as,

$$
C_{m}=\frac{\text { Moment }}{0.5 \rho V^{2} S r}
$$

where $\rho$ was the water density (set to $1000 \frac{\mathrm{kg}}{\mathrm{m}^{3}}$ for all simulations), $S$ was the turbine frontal area, and the Moment generated by the turbine was taken from CFD or EFD results.

\subsection{Computational Domain and Boundary Conditions}

The boundary conditions used for all CFD studies are outlined in Table 2 for the boundaries shown in Figure 3. The boundaries conditions simulated free stream conditions ensuring that the turbine was isolated from any boundary layer or blockage effects and allowed full wake development. Domain symmetry was used to reduce overall mesh size by splitting the domain along the horizontal centre plane, with the resultant half domain used for all simulations. No free surface effects were simulated, as the turbine was assumed to operate at sufficient depth to minimise any surface effects. 
Table 2: Domain boundary conditions for CFD models

\begin{tabular}{cc}
\hline Wall & Boundary condition \\
\hline Inlet & Uniform flow: $1.5 \mathrm{~ms}^{-1}$ \\
Outlet & Pressure: $0 \mathrm{~Pa}$ \\
Walls & Free slip walls \\
Turbine & No slip walls \\
Symmetry & Symmetry walls
\end{tabular}

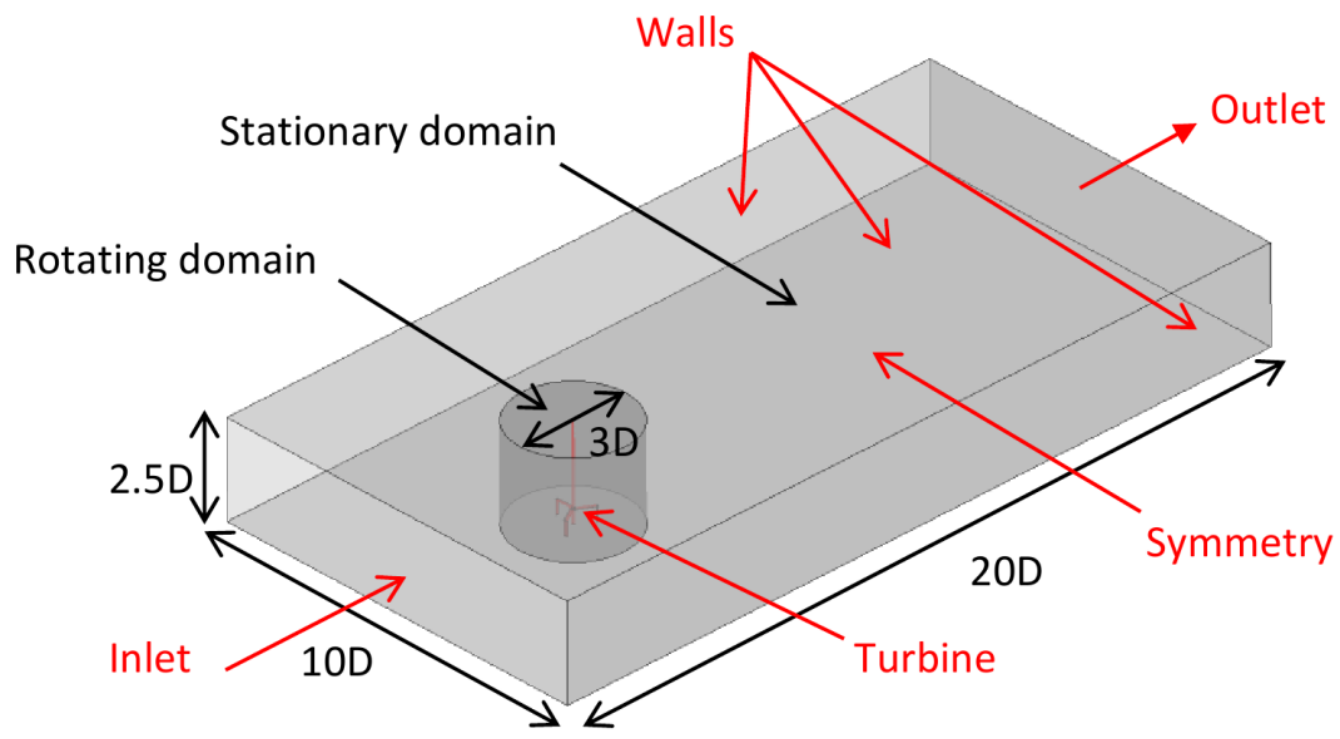

Figure 3: Domain boundary nomenclature and sizing, dimensions in turbine diameters D

The CFX transient rotor-stator model was utilised to simulate rotation at each time step, with the rotation interface modelled using a General Grid Interface (GGI). The GGI method placed an interface between the stationary outside domain and the rotating inner domain, shown in Figures 3 and 4, allowing flow values to be calculated on either side of the boundary by an intersection algorithm [19]. This GGI interface was set at a distance of 1.5 times the turbine diameter, D, from the turbine rotation axis, and mesh density was increased on the interface to limit interpolation errors on $C_{p}$ predictions. For all simulations the inner domain was rotated at the desired $\lambda$, and for validation purposes this corresponded with the relevant EFD rotational rates. 


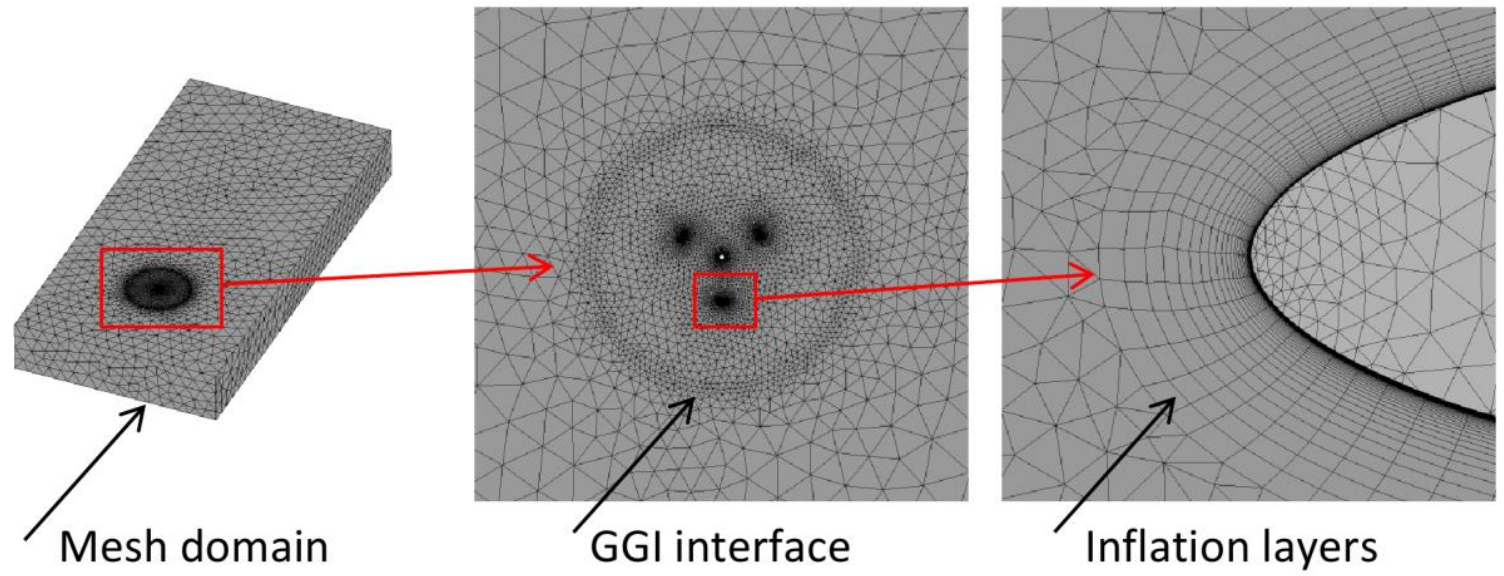

Figure 4: Mesh domain showing overall mesh domain, GGI interface, and inflation layer detail

All CFD meshes were generated with ANSYS CFX 13.0 mesher [21] using unstructured tetrahedral elements. All 3D geometrical features, including all blades, hubs, shafts, struts and blade-strut joints were modelled, with the main mesh features shown in Figure 4. Mesh density was refined by specifying face sizing, cell curvature angle and expansions rates on areas of interest, such as on blades, struts, and the turbine wake to fully capture flow hydrodynamics, with inflation layers utilised to control cell heights near all surfaces to resolve the boundary layers. Conversely, the density of the mesh was reduced in regions such as the boundary fields where a coarse mesh was found sufficient.

\subsection{Turbulence Model and Discretisation Schemes}

The k- $\omega$ SST turbulence model was selected as it has previously been successfully used to simulate turbine performance $[3,8,14,18,22,23]$ due to its ability to model both the boundary layer and the free stream regions, as well as offering improved prediction of flow separation and adverse pressure gradients by the inclusion of transport effects into the formulation of the eddy-viscosity [19,24]. The height of the first cell layer on all turbine surfaces was specified to ensure that it was within the viscous sub-layer, with resultant average $y+$ (the dimensionless distance from the wall) values less than 1. Simulations using reduced boundary layer inflation density and thus higher $y+$ values resulted in reduced simulation accuracy, as the ANSYS-prescribed wall functions [19] were unable to resolve the flow near the walls due to high levels of separation and adverse pressure gradients. All simulations were performed using a high order advection and second order backwards Euler transient scheme. 
To reduce simulation time, simulations were initialised using previous solutions and were deemed completed when the magnitude of torque fluctuations over each revolution reached a quasi-steady state, usually taking 2-3 revolutions. Convergence was achieved when residuals converged to within $10^{-4}$ for each time step and reduced by more than three orders of magnitude. An inlet turbulence intensity level of $5 \%$ was used for all simulations as commonly performed $[3,8,15,17]$, as EFD data was not available.

\subsection{Grid Independence Studies}

Studies of the influence of grid resolution factors, including mesh density, time step size, domain length, width, height and domain symmetry were conducted to ensure grid independence. These investigations were performed both quantitatively, by examining relationships between mesh grid resolution and $C_{p}$, as well as qualitatively, with graphical methods used to evaluate any changes between grid resolution factors. Quantitative independence was deemed satisfactory when changes in grid parameters resulted in $C_{p}$ differences of less than $5 \%$, resulting in a suitable balance between solution independence, speed and computational effort.

\subsubsection{Mesh Density Independence}

Mesh density independence was evaluated for five densities, shown in Figure 5, with independence demonstrated for turbine $\mathrm{A}$ at a minimum mesh density of $5 \times 10^{-4} \mathrm{~m}$, corresponding to 17.2 million elements, with a visual comparison of mesh density shown in Figure 6. Predictions of $C_{p}$ were sensitive to changes in mesh density, with increased mesh resolution on blade surfaces needed to capture the complex flow structure. Using similar methods, mesh density independence for turbines B and $\mathrm{C}$ was determined corresponding to 17.3 million and 16.6 million elements respectively. 


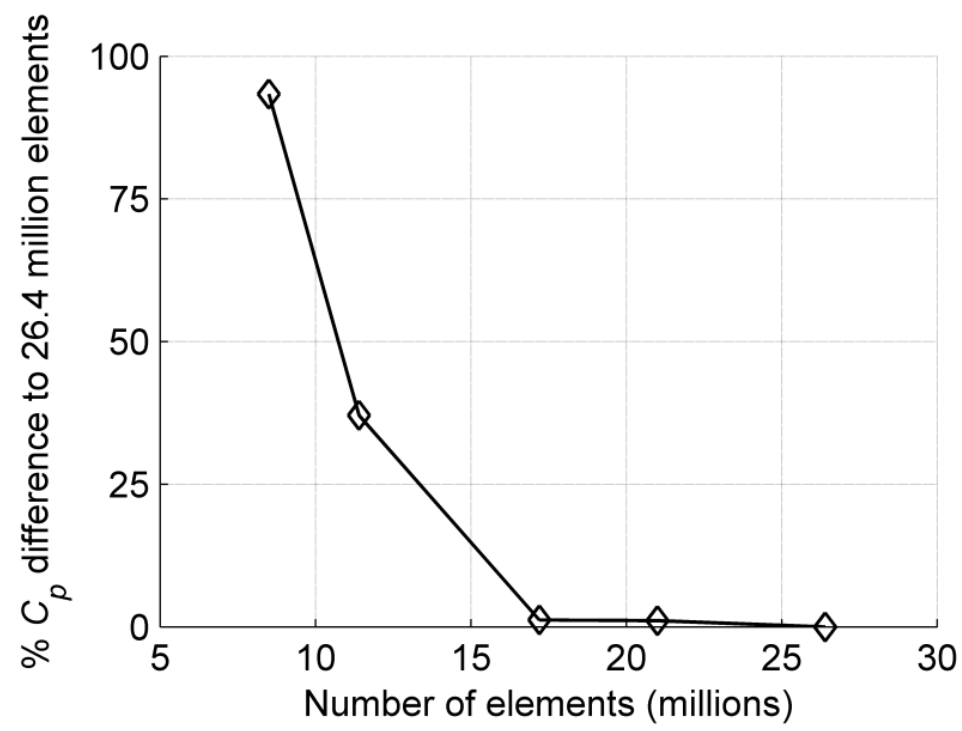

Figure 5: Mesh density independence study for turbine A at an inflow velocity of $1.5 \mathrm{~ms}^{-1}$ and $\lambda=2.75$

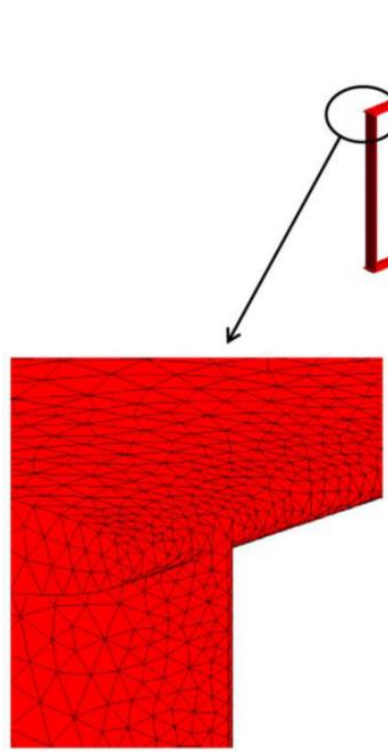

a

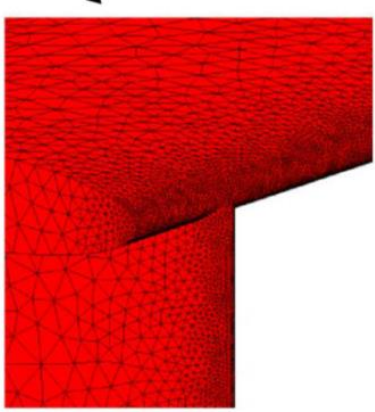

b

Figure 6: Comparison of mesh density on surfaces of blades and struts between (a) 8.5 million and (b) 27 million elements

\subsubsection{Time Step Independence}

Time step studies were performed to ensure temporal $C_{p}$ independence, critical due to the highly transient nature of the flow. Simulations were performed for turbine A using time steps from $0.225^{\circ}$ to $3.6^{\circ}$ of turbine rotation per step with 17.2 million elements, with independence determined at a time 
step of $0.9^{\circ}$ rotation per step, as shown in Figure 7. Using similar methods time step independence for turbines $\mathrm{B}$ and $\mathrm{C}$ was found at $0.9^{\circ}$ rotation per step.

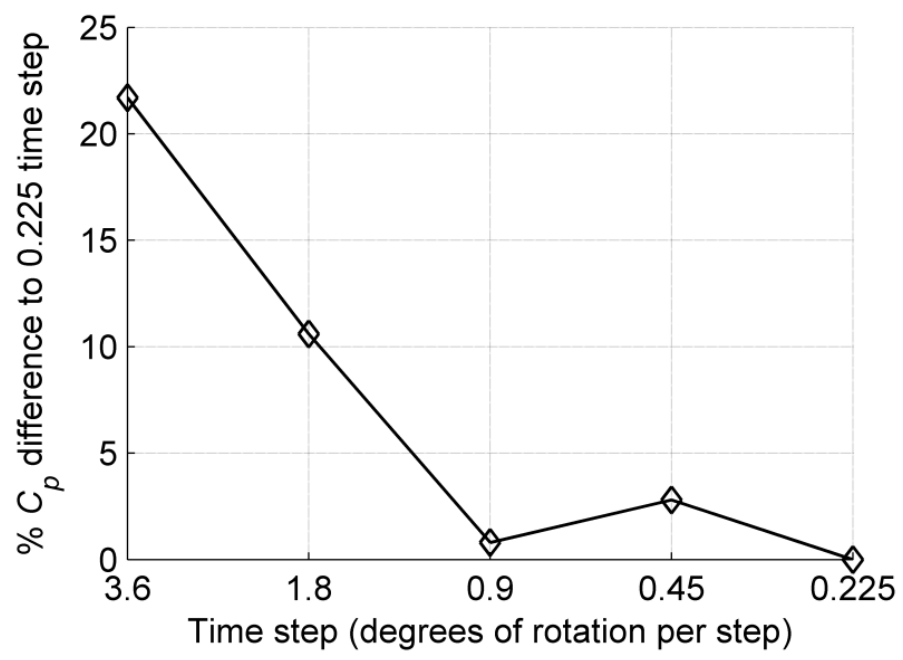

Figure 7: Time step independence study for turbine $A$ at an inflow velocity of $1.5 \mathrm{~ms}^{-1}$ and $\lambda=2.75$

\subsubsection{Domain Size Independence}

Domain size independence studies were performed to ensure that the turbine was isolated from any domain wall or blockage effects and to allow full wake development, with changes in simulation domain length, width, and height. Results for turbine A, shown in Table 3, indicate that a domain length of $20 \mathrm{D}$, width of $10 \mathrm{D}$ and height of $2.5 \mathrm{D}$, with the turbine located $5 \mathrm{D}$ from the inlet, allowed full wake development whilst minimising domain wall and blockage effects. Qualitatively domain wall height effects are shown in Figure 8, where the proximity of the wall to the turbine due to low domain height of $1.25 \mathrm{D}$ increased flow velocity through the domain due to flow constriction, artificially increasing $C_{p}$. Although not studied here this constriction effect could be harnessed to increase $C_{p}$ through the use of shaped ducts or walls or limited water depths [12].

Table 3: Domain size independence study for turbine $A$ at $1.5 \mathrm{~ms}^{-1}$ and $\lambda=2.75$

\begin{tabular}{|c|c|c|c|c|c|c|c|c|}
\hline $\begin{array}{l}\text { Domain } \\
\text { Length } \\
\text { (D) }\end{array}$ & $C_{p}$ & $\begin{array}{c}\% C_{p} \\
\text { change } \\
\text { from 20D } \\
\text { case }\end{array}$ & $\begin{array}{c}\text { Domain } \\
\text { Width } \\
\text { (D) }\end{array}$ & $C_{p}$ & $\begin{array}{c}\% C_{p} \\
\text { change } \\
\text { from } 10 \mathrm{D} \\
\text { case }\end{array}$ & $\begin{array}{c}\text { Domain } \\
\text { Height } \\
\text { (D) }\end{array}$ & $C_{p}$ & $\begin{array}{c}\% C_{p} \\
\text { change } \\
\text { from } 2.5 \mathrm{D} \\
\text { case }\end{array}$ \\
\hline 5 & 0.285 & $11.3 \%$ & 5 & 0.267 & $4.3 \%$ & 1.25 & 0.276 & $7.8 \%$ \\
\hline 20 & 0.256 & - & 10 & 0.256 & - & 2.5 & 0.256 & - \\
\hline 40 & 0.256 & $0.0 \%$ & 20 & 0.251 & $2.0 \%$ & 5 & 0.249 & $2.7 \%$ \\
\hline
\end{tabular}




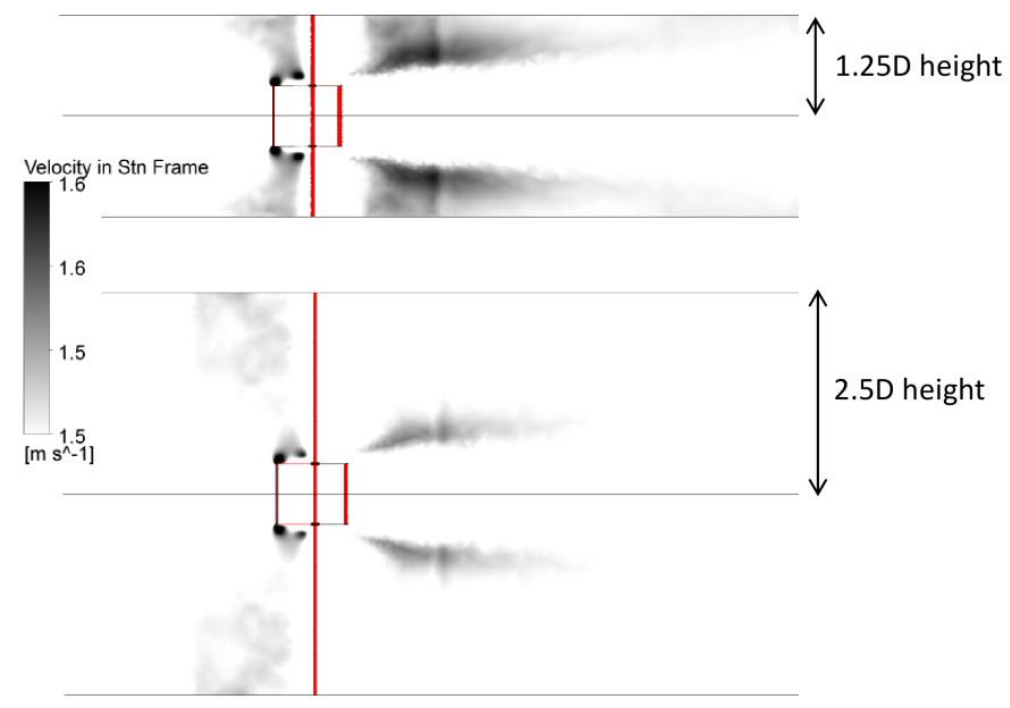

Figure 8: Comparison of flow velocity for domain heights of $1.25 \mathrm{D}$ and $2.5 \mathrm{D}$ showing increase in velocity due to flow constriction, turbine $A$ at an inflow velocity of $1.5 \mathrm{~ms}^{-1}$ and $\lambda=2.75$

\subsubsection{Domain Symmetry Validation}

In order to validate the use of the half domain split along the horizontal mid plane, equivalent simulations were carried out on full and half domains, with results shown in Table 4, with differences in $C_{p}$ of less than $0.4 \%$ between the two domains. Velocity profiles for both the half and full domain, shown in Figure 9, reveal minimal difference in velocity distribution, allowing the use of the half domain, thereby reducing overall mesh size by two.

Table 4: Comparison of $C_{p}$ for symmetrical and full domains at an inflow velocity of $1.5 \mathrm{~ms}^{-1}$ and $\lambda=2.75$

\begin{tabular}{ccc}
\hline Domain & Symmetrical domain & Full domain \\
\hline $\boldsymbol{C}_{p}$ & 0.256 & 0.255 \\
$\% \boldsymbol{C}_{p}$ change from & - & $0.4 \%$
\end{tabular}

symmetrical domain 


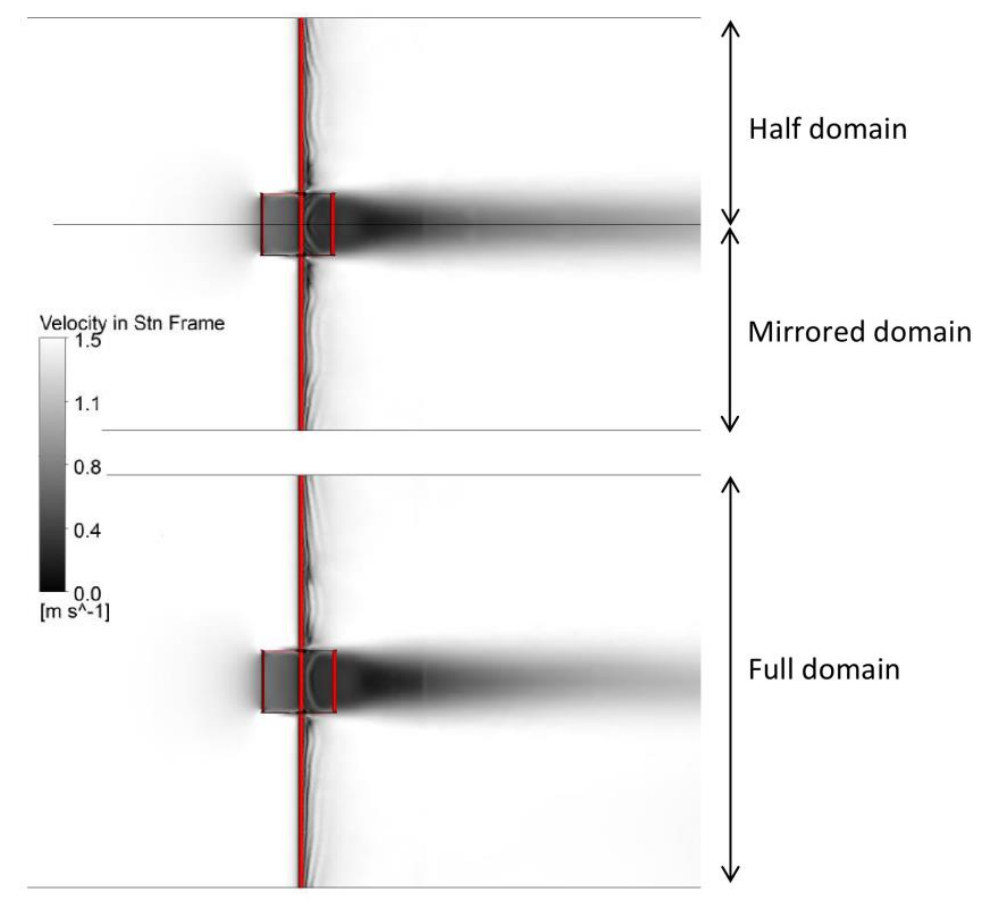

Figure 9: Velocity in stationary frame comparisons for half domain and full domain for turbine A at an inflow velocity of $1.5 \mathrm{~ms}^{-1}$ and $\lambda=2.75$

\subsection{Numerical Simulation Time}

Numerous authors $[11,13,18]$ have suggested that 3D CFD models are not practical for turbine performance investigations due to their excessive simulation time and computational requirements. However full 3D simulations are now feasible due to increases in cluster power and the increased efficiency of distributed CFD solutions, with $C_{p}$ simulations for one revolution at each $\lambda$ taking 24 hours on an 18 core cluster comprising of Intel Core 2 Quad Q9300 processors with 2GB memory per core, allowing the simulation of vertical axis turbine performance in a timely and efficient manner. All simulations were performed using URANS models, which combined with free surface modelling assumptions minimised simulation time when compared to numerical methods such as LES,

\section{Results and Discussion}

Simulations of power output, torque fluctuations and mounting forces were obtained for turbines A, B and $\mathrm{C}$ at an inflow velocity of $1.5 \mathrm{~ms}^{-1}$ for varying rotational rates, using the simulation settings outlined in Table 5. 
Table 5: Main CFD simulation settings

\begin{tabular}{cc}
\hline CFD & Setting \\
\hline Turbulence model & k- $\omega$ SST \\
Time step & $0.9^{\circ}$ rotation per time step \\
Advection scheme & High order \\
Transient scheme & Second order backwards Euler \\
Domain length & $20 \mathrm{D}$ \\
Domain width & $10 \mathrm{D}$ \\
Domain height & $2.5 \mathrm{D}$
\end{tabular}

\subsection{Validation of Numerical Simulations with Experimental Fluid Dynamics}

Validation studies were performed against EFD tests at the University of British Columbia's towing tank, an approximately $60.1 \mathrm{~m}$ long, $3.7 \mathrm{~m}$ wide and $2.4 \mathrm{~m}$ deep facility [20]. Using a torque sensor and rotation rate encoder, $C_{p}$ for varying $\lambda$ were obtained at a series of flow velocities from $0.75 \mathrm{~ms}^{-1}$ to $2.24 \mathrm{~ms}^{-1}$, with $\lambda$ varied using a motor drive unit through a 20:1 gearbox. Shaft force was measured using two load cells mounted to the vertical shaft. The turbine models A and B simulated in this project were based on two of the EFD turbines tested by Rawlings [20], enabling the equivalent CFD simulations to be validated.

Comparisons of CFD and EFD $C_{p}-\lambda$ curves for turbines A and B are shown in Figure 10. At low $\lambda$, good agreement was found between CFD and EFD results for both turbines. Below $\lambda=3$ all turbine A results were within EFD error bars, with $C_{p}$ differences between CFD and EFD results of $17 \%$ and $0.8 \%$ at $\lambda=1.5$ and $\lambda=2.5$ respectively. Turbine B prediction accuracy at low $\lambda$ was similar, with differences in $C_{p}$ prediction of $29.2 \%, 14.4 \%$ and $1.7 \%$ at $\lambda=1.5,2$ and 2.25 respectively. However, for both turbines $C_{p}$ prediction accuracy reduced as $\lambda$ increased past the location of maximum $C_{p}$, with CFD $C_{p}$ values shifted lower. The authors suggest that this was due to over prediction of turbine blade airfoil drag due to the use of a fully turbulent CFD model, which over-estimated skin friction and hence airfoil drag [25] particularly at low angles of attack, reducing $C_{p}$ at high $\lambda$ where blade angles of attack are low [7]. Improvements of simulation accuracy at high $\lambda$ may be possible using newly developed transitional turbulent models that can account for this laminar-turbulent transition behaviour [19]. Over prediction of $C_{p}$ at high $\lambda$ may also occur as a result of blockage errors that were not accounted for in EFD results which could artificially increase EFD $C_{p}$ [26], and may also occur due to differences in the turbulence intensity levels between the CFD models and EFD testing, as high 
turbulence intensity levels can delay stall [27], leading to increases in $C_{p}$ especially at high $\lambda$ [28], with no turbulence intensity measurements recorded during EFD testing to compare to CFD turbulence levels.



Figure 10: Comparison of CFD and EFD $C_{p}-\lambda$ curves for turbines A and B at an inflow velocity of 1.5 $\mathrm{ms}^{-1}$. Error bars only reported for EFD turbine A [20]

Significantly both CFD models were able to accurately capture the effect of geometrical changes on maximum $C_{p}$, which was simulated to within $14.3 \%$ and $6.3 \%$ of EFD results for turbines A and B respectively. This prediction accuracy is much higher than previous vertical axis turbine CFD predictions, which exhibit maximum $C_{p}$ errors of more than $45 \%$ [12,14,29-31], possibly due to the inclusion of the full turbine geometry in the present study. The use of 3D models also allows for the direct simulation of $C_{p}$ without the need for empirical correction for 2D models as previous used $[8,10,16]$.

It has previously been suggested that URANS methods are unable to accurately predict vertical axis turbine blade vortex shedding and flow diffusion, requiring higher order CFD methods such as Large Eddy Simulation (LES) [13]. However the authors believe that the accuracy of 3D CFD simulations when compared to EFD results, as demonstrated in Figure 10, suggests that reasonable estimates of performance coefficients such as $C_{p}$ can be obtained by URANS methods and that resolution of smallscale flow field detail by LES or Direct Numerical Simulation (DNS) methods may not be necessary. Previous URANS $C_{p}$ prediction errors of more than $45 \%$ [12,14,29-31] may be influenced more by 
their 2D nature than overall CFD approach, with the lack of strut and tip effects having a greater influence on $C_{p}$ predictions than poor fine-scale vortex resolution. Validation of this hypothesis could be performed by comparing flow field simulations with EFD using Particle Image Velocimetry (PIV) to establish the accuracy of the simulated vortex shedding and flow diffusion effects and their influence on turbine performance characteristics [32]

\subsection{Numerical Simulation of Power Output}

Using the validated CFD simulation methods $C_{p}-\lambda$ curves for turbines $\mathrm{A}, \mathrm{B}$ and $\mathrm{C}$ were determined, and are shown in Figure 11. Although the turbines were the same in overall geometrical dimensions, there were significant $C_{p}$ variations between the turbine designs for all $\lambda$ as a result of the changes in strut section, blade-strut joint design and strut location.

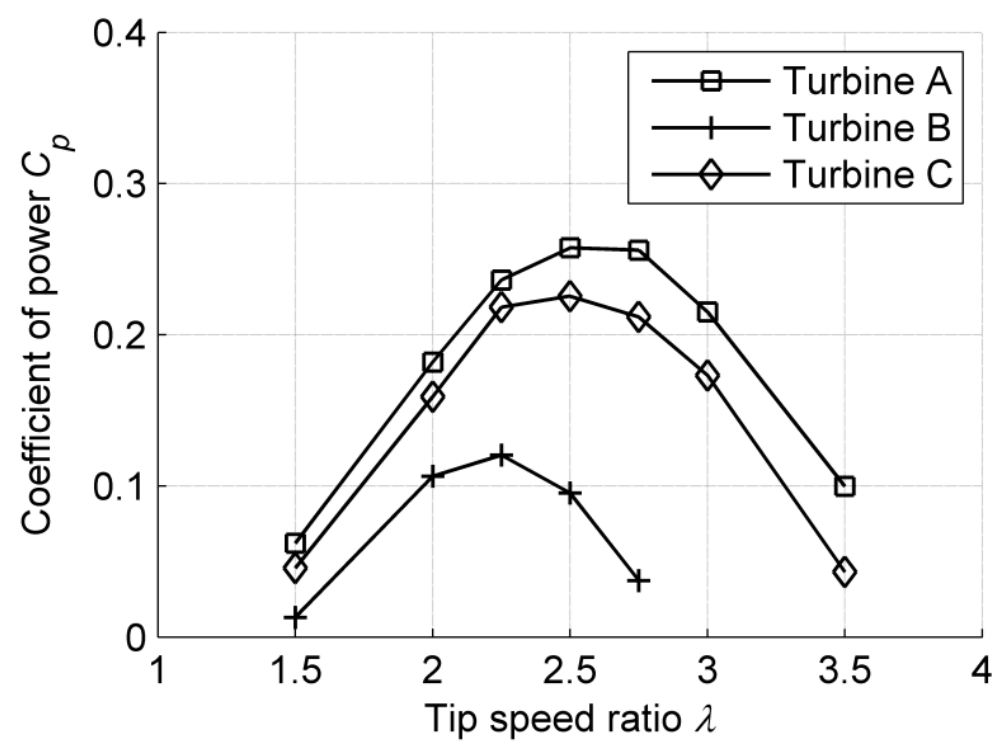

Figure 11: Comparisons of CFD $C_{p}-\lambda$ curves for turbines A, B, and C at an inflow velocity of $1.5 \mathrm{~ms}^{-1}$

\subsubsection{Influence of Strut Section and Blade-Strut Joint Design}

The influence of strut section and blade-strut joint design on $C_{p}$ was found to be significant, as shown in Figure 11. The $C_{p}$ for turbine $\mathrm{C}$ is more than double that of turbine B. This large increase in $C_{p}$ is due to a change in strut section and blade-strut joint design, with all other geometry being identical for the two turbines. As shown in Figure 2, turbine $\mathrm{C}$ has a NACA0012 section for the strut and faired 
joints with the blades, whereas the turbine B struts have a machined flat bar section and small connection tabs for the blade-strut joints. The more streamlined hydrodynamic strut section of turbine C provides lower levels of strut drag than the bluffer section used for turbine B, resulting in reduced levels of resistive torque generated by the turbine struts. This influence can be seen in Figure 11 to increase as the tip speed ratio increases. The mounting tabs located at the blade-strut joints used in turbine B generated higher levels of parasitic drag when compared to the more streamlined design of turbine $\mathrm{C}$, thus contributing to the $C_{p}$ reduction. Although it is difficult to isolate whether the strut section or the blade-strut joint design had the largest impact on $C_{p}$, combined their effect on turbine performance was significant.

These outcomes from the CFD study are supported by the EFD results, where significant changes in $C_{p}$ were found and attributed to strut drag and blade-strut joint changes between the two designs [20]. The results show that to maximise $C_{p}$ the selection of strut section and blade-strut joint design is critical. If optimisation studies are performed solely on blade section, as would occur using 2D CFD models, the effects on $C_{p}$ of strut section and blade-strut joint design would not be captured.

\subsubsection{Influence of Strut Location}

The location of the turbine struts was found to impact on $C_{p}$, with struts located at blade tips increasing effective blade length and acting as end-plates, increasing maximum $C_{p}$ by $12.4 \%$ as shown in Figure 11 when comparing turbines $\mathrm{A}$ and $\mathrm{C}$ with strut locations at blade tips and quarter span respectively. Although the increase $C_{p}$ was less than that caused by strut section and blade-strut joint changes it was still considerable. This end plate effect was also found in EFD where similar $C_{p}$ increases of up to $16 \%$ at $1.5 \mathrm{~ms}^{-1}$ were found for turbine B using NACA shaped end plates and circular disks $[20,33]$.

Increases in $C_{p}$ caused by increased effective blade length can be seen in the differences between the vortex structures shown in Figure 12. Turbine $\mathrm{C}$ generated vortex structures at both the blade ends and the quarter span, whereas the location of turbine A struts at the blade tips combined the tip and bladestrut joint vortex structures, minimising flow disturbance over the blade and thereby increasing total lift force and thus torque and $C_{p}$ generated. 


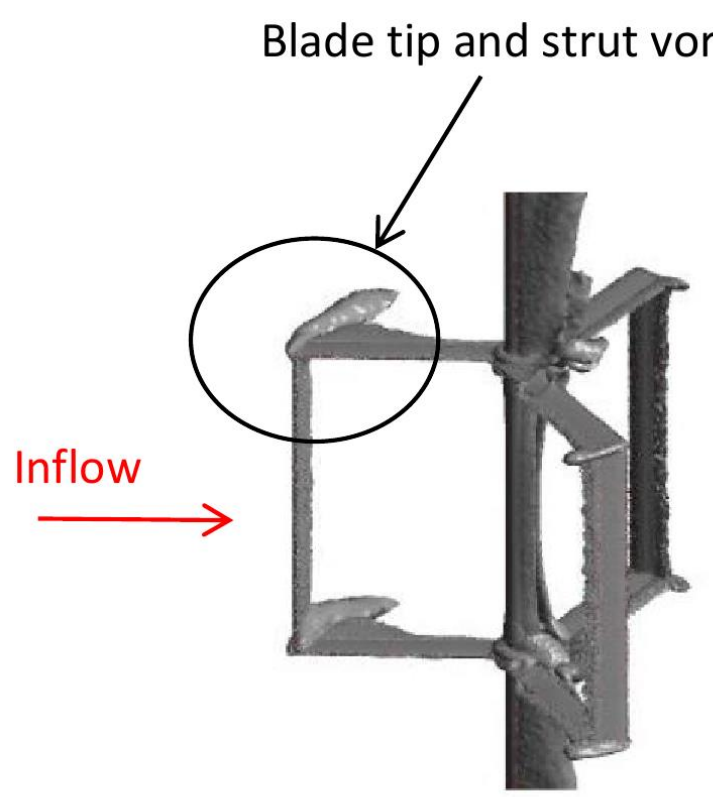

Turbine A

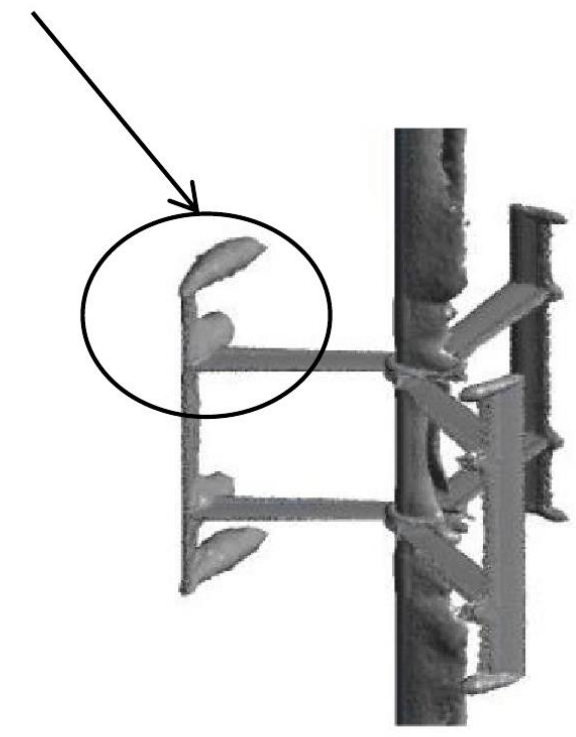

Turbine $\mathrm{C}$

Figure 12: Blade tip and strut vortex structures for Turbine A and C, vorticity in stationary frame of $25 \mathrm{~s}^{-1}$ at an inflow velocity of $1.5 \mathrm{~ms}^{-1}$ and $\lambda=2.75$

\subsection{Vortex Shedding Visualisation}

Comparisons of vortex shedding strength were performed for $\lambda=1.5$ and $\lambda=2.75$ for turbine $\mathrm{B}$, as shown in Figure 13. The influence of $\lambda$ on vortex shedding as a result of the relationship between blade angles of attack and $\lambda$ [7] can be seen. At $\lambda=1.5$, large vortices were shed from the blades, as they stalled in both the upstream and downstream turbine sections due to the high angles of attack experienced at low $\lambda$. Vortices shed from the blades traverse downstream with the water flow, impacting on the blades as they rotate, reducing blade efficiency and thus the torque generated. However, at $\lambda=2.75$ there was a marked reduction in vortex generation due to the high turbine rotational rate relative to the inflow velocity. This results in the blades fluctuating through lower angle of attack ranges [7], reducing flow separation and hence vortex generation, and thus increasing blade efficiency and torque generated. Shaft vortex shedding at $\lambda=1.5$ can also be seen to impact on the downstream blades, however at $\lambda=2.75$ again this effect was greatly reduced, as the increase in rotation speed of the cylindrical shaft reduces the intensity of vortex shedding [34]. Validation of these vortex shedding simulations was not possible as EFD results included no flow visualisation, although validation would be possible if EFD techniques such as PIV were performed. 


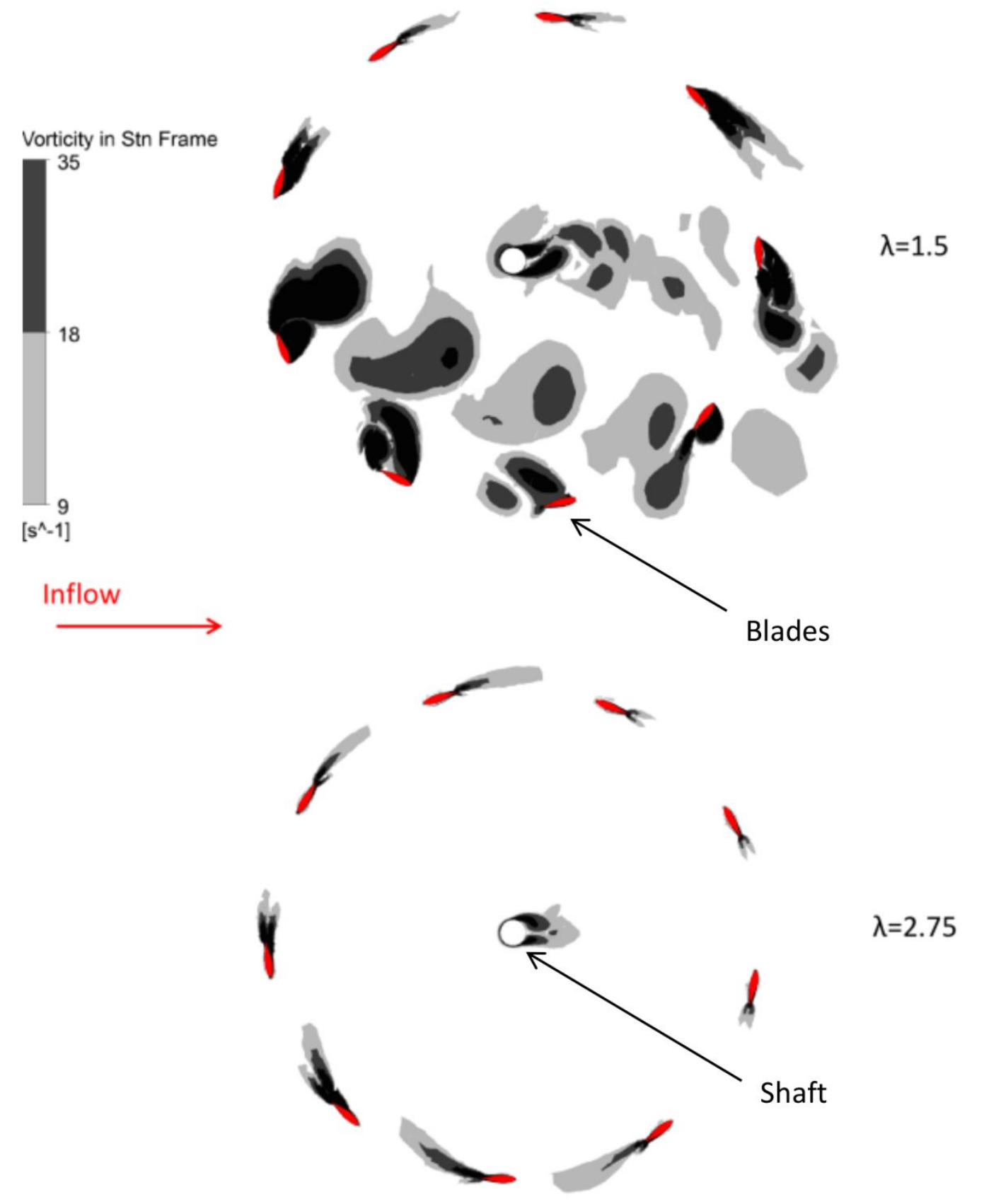

Figure 13: Vortex structure visualisation for turbine $B$ at $\lambda=1.5$ and $\lambda=2.75$ showing vortex generation. Time series of isosurfaces of vorticity in stationary frame from 9 to $35 \mathrm{~s}^{-1}$ at an inflow velocity of $1.5 \mathrm{~ms}^{-1}$ on plane $0.05 \mathrm{~m}$ from horizontal symmetry

\subsection{Torque Ripple Simulations}

The cyclic nature of the forces acting on the blades caused by the changing angles of attack resulted in fluctuations in torque generated. These time variations in torque are transmitted through the turbine 
drive train and can cause shaft vibrations and potentially damage turbine components [35-36]. Various methods for quantifying torque fluctuations have previously been used [11,16,35-37]; in this work torque ripple is determined as a Torque Ripple Factor $(T R F)$, where,

$$
T R F=C_{m_{\text {Max }}}-C_{m_{\text {Min }}}
$$

where $C_{m_{\text {Max }}}$ and $C_{m_{\text {Min }}}$ are the maximum and minimum moment coefficients, as demonstrated in Figure 14 for turbine $A$ at $\lambda=2.75$. This formulation allows for easy comparison of the range of torque variations experienced and is similar to that used by Winchester and Quayle [36] and Shiono et al. [37]

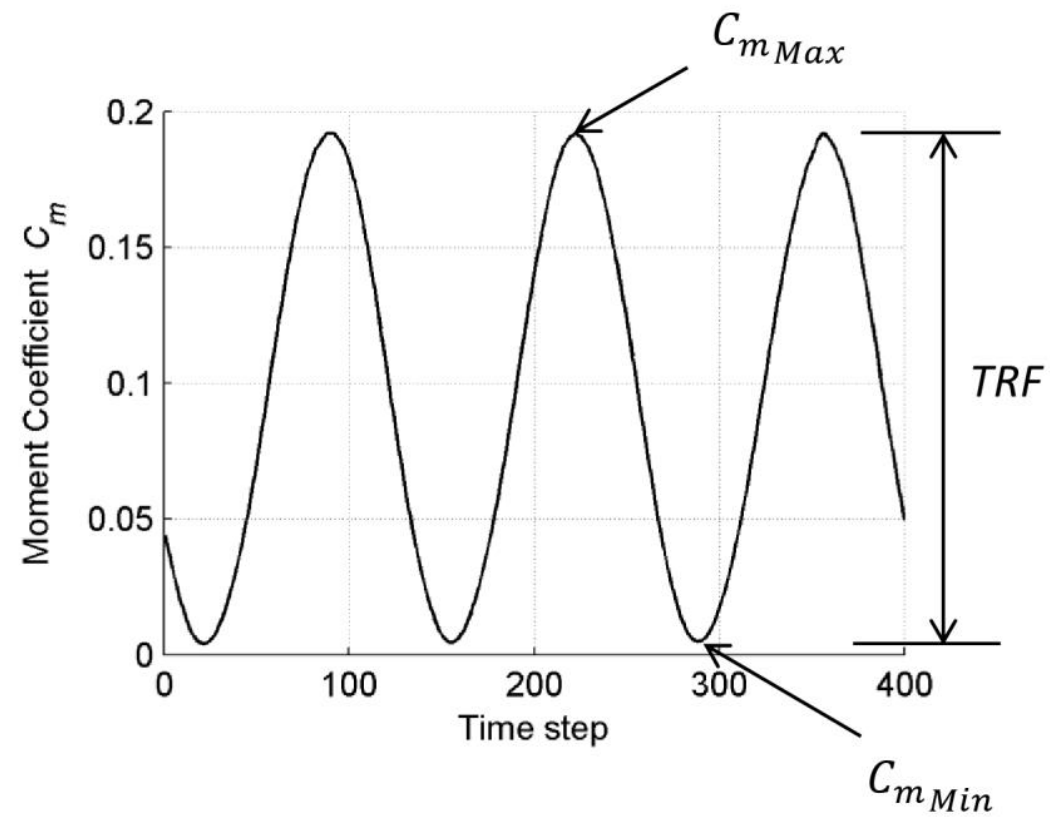

Figure 14: Torque Ripple Factor (TRF) determination for turbine A at an inflow velocity of $1.5 \mathrm{~ms}^{-1}$ and $\lambda=2.75$

The maximum TRF for each of the three turbines occurred slightly below the $\lambda$ location of maximum $C_{p}$, as shown in Figure 15, which for turbines A, B, and C were located at $\lambda=2,2$, and 2.25 respectively. This was due to the forces on the blades alternating between high positive and negative values of lift in the upstream and downstream areas of rotation. Maximum TRF was found to increase with turbine efficiency, with turbine A showing increases in $T R F$ of $53.7 \%$ and $8.7 \%$ compared to turbines $\mathrm{B}$ and $\mathrm{C}$ respectively, due to the increase in total lift forces. Both the location of maximum 
$T R F$ and its relationship with $C_{p}$ are unfortunate, as turbines designed to operate at maximum $C_{p}$ will operate near maximum $T R F$, which can significantly shorten turbine life due to the large alternating loading forces and thus fatigue [35,36]. Reductions of $T R F$ would be possible by using helical bladed turbines, as the helical blade inclination angle reduces torque pulsation [37].

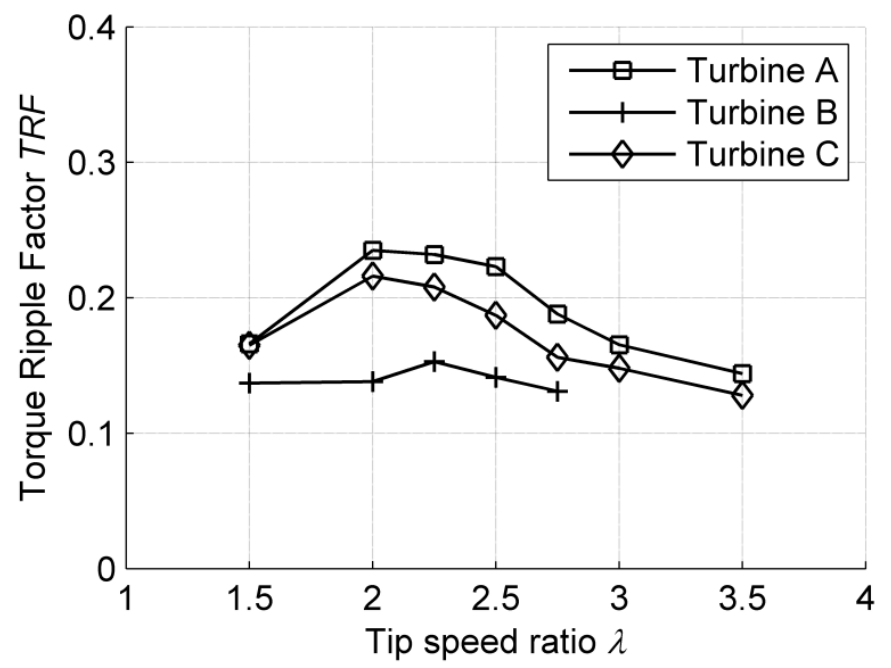

Figure 15: Comparison of CFD Torque Ripple Factor $(T R F)$ for turbines A, B, and C at an inflow velocity of $1.5 \mathrm{~ms}^{-1}$

\subsection{Turbine Mounting Force Simulations}

Turbine loading forces in the inline, lateral and vertical direction shown in Figure 16 were simulated, with no force in the vertical direction found due to the horizontal symmetry of the turbine design. Both average and maximum forces were evaluated as the loading forces vary as the turbine rotates. The inline, lateral and maximum forces were non-dimensionalised as,

$$
\begin{array}{r}
F_{I}=\text { Inline Force } / \frac{1}{2} \rho V^{2} S \\
F_{T}=\text { Lateral Force } / \frac{1}{2} \rho V^{2} S \\
F_{\text {Max }}=\text { Maximum Force } / \frac{1}{2} \rho V^{2} S
\end{array}
$$




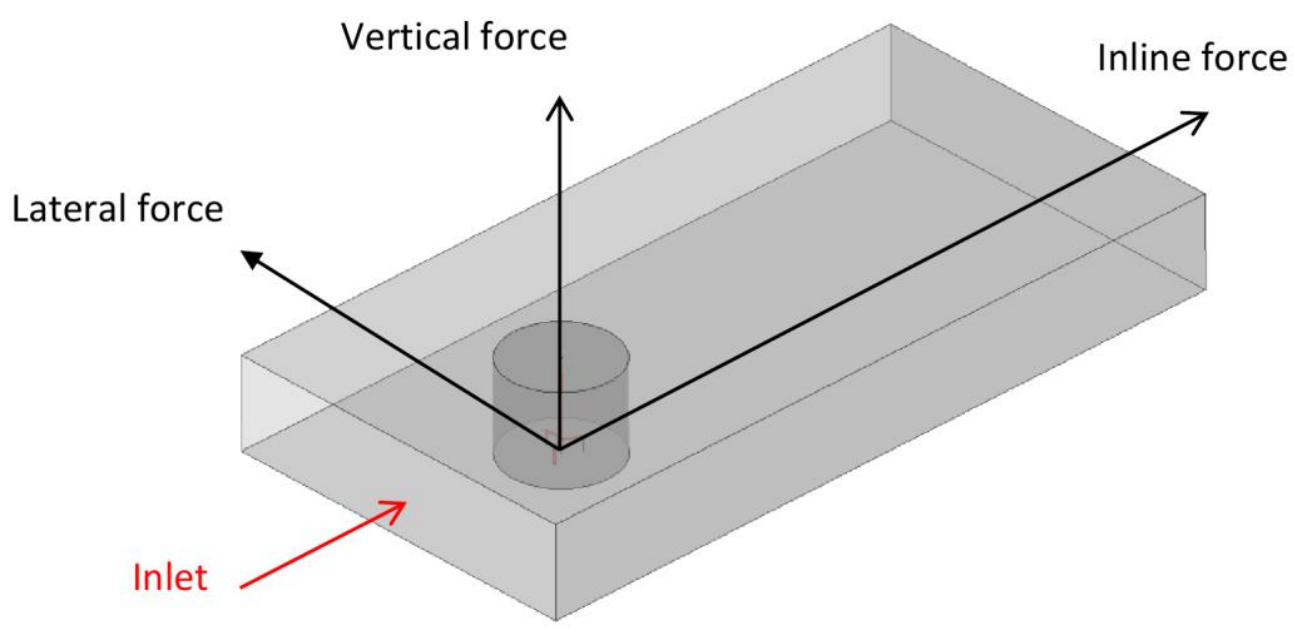

Figure 16: Definitions of lateral, inline and vertical forces

\subsubsection{Validation of Inline Forces with Experimental Fluid Dynamics}

Average CFD and EFD [20] inline force coefficients for turbine B are shown in Figure 17. Good agreement between CFD and EFD was found across most $\lambda$, with differences of $8.2 \%$ and $2.5 \%$ at $\lambda=2$ and $\lambda=2.5$ respectively. The inline force magnitude increased with $\lambda$ due to increased flow velocity over the struts and hence increased strut drag. However the inline force plateaued with increasing $\lambda$, which the authors suggest is caused by reductions in cylindrical shaft drag coefficient as $\lambda$ increases, as shown by EFD and CFD studies on cylindrical bodies [38]. This effect may also be caused by the turbine acting more like a solid body at high $\lambda$ [39]. Validation of lateral, vertical and maximum forces was not possible as EFD data was not available however close correlation between EFD and CFD was demonstrated for inline force coefficients simulations. 


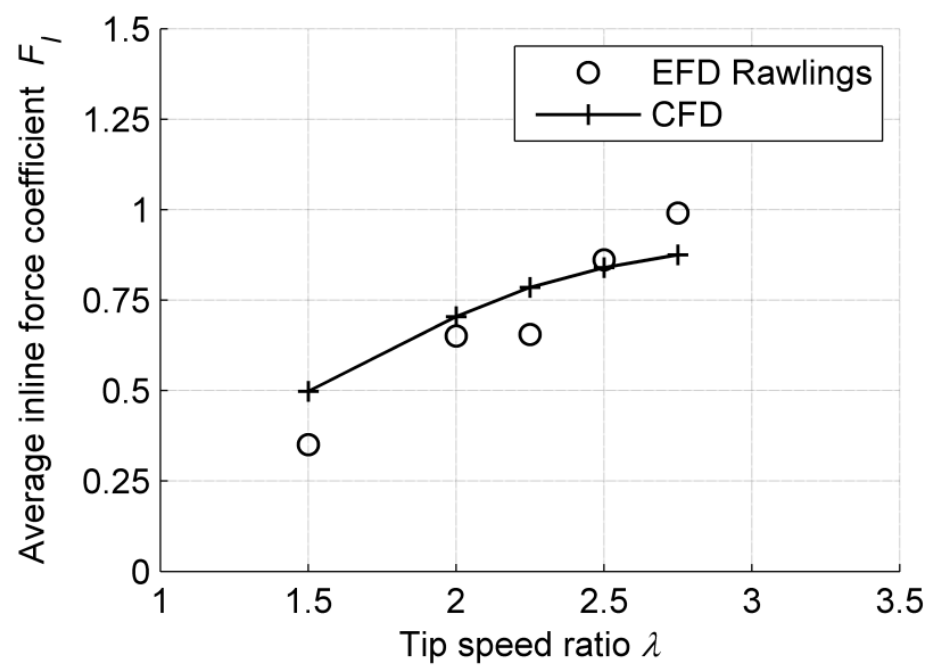

Figure 17: Comparison of average CFD and EFD inline force coefficients for turbine B at an inflow velocity of $1.5 \mathrm{~ms}^{-1}$

\subsubsection{Inline and Lateral Force Simulations}

Simulations of inline and lateral force coefficients for turbines A, B, and C were obtained and are shown in Figure 18. Lateral forces were found to be approximately $75 \%$ lower than inline forces, and were found to remain relatively constant across the $\lambda$ range. Inline force rose then plateaued as $\lambda$ increased, due to reductions in shaft drag as rotation rates increased. Notably inline forces were not significantly influenced by strut section, blade-strut joint design or strut location, whereas the lateral forces were affected by changes in both parameters due to variations in blade efficiency and thus blade lift force between turbine A, B and C. The maximum forces generated were found to be up to $40 \%$ higher than the average forces due to TRF (Eqn. 4), with the average loading forces slowly plateauing above $\lambda=3$ due to reductions in shaft drag and changes in blockage as $\lambda$ increased. Below $\lambda=3$, maximum mounting forces at each $\lambda$ increased with turbine efficiency due to lateral force increases, with variations in lift force driving the difference. 


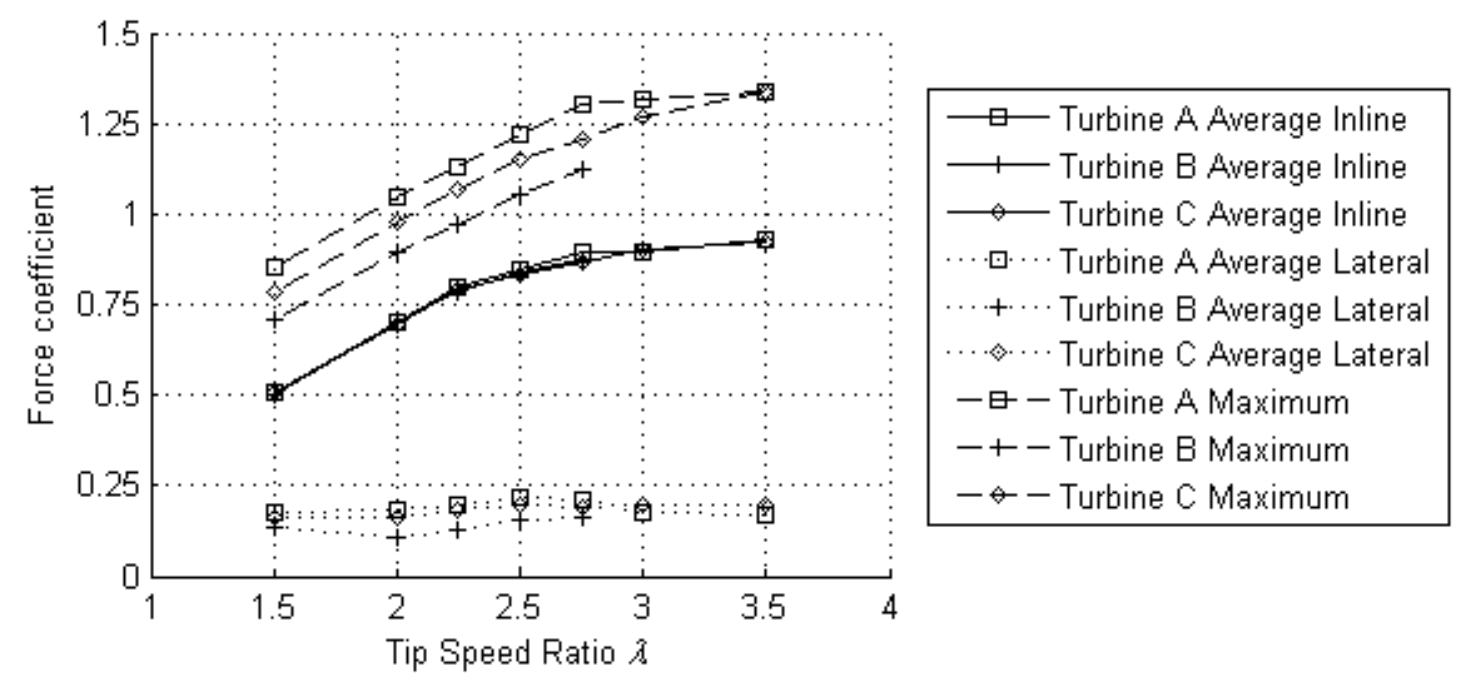

Figure 18: Comparisons of average inline, average lateral and maximum force coefficients for turbines $\mathrm{A}, \mathrm{B}$, and $\mathrm{C}$ at an inflow velocity of $1.5 \mathrm{~ms}^{-1}$

\section{Conclusions}

Three straight-bladed vertical axis tidal turbine configurations were simulated successfully by $3 \mathrm{D}$ CFD models using the SST turbulence model. Two turbine models were successfully validated against EFD results, with maximum power output predictions within 15\% of EFD results. These results demonstrate that URANS models are an effective simulation tool for predicting vertical axis turbine power output, and that they can accurately capture the effects of geometrical changes on power output between turbine designs.

The selection of strut section and blade-strut joint design was found to have a crucial impact on power output. Power output with low drag struts with streamlined blade-strut joints was found to be more than $50 \%$ higher than that of high drag struts with blade-strut connection tabs. The location of the strut attachment point to the blade was found to impact power output to a lesser but still significant degree, with struts located at the blade tips increasing maximum power output by $12 \%$ when compared to struts located at the quarter span. 
The relationship between torque ripple and rotation rate was investigated, with maximum torque ripple occurring around the rotation rate where maximum power output was generated. As turbines are designed to operate at maximum power output they will experience large loading fluctuations, the effects of which need to be further investigated

Investigation of mounting forces using CFD models determined new relationships between rotational rate and mounting forces. The average inline force magnitude was found to be significant, with average lateral forces exhibiting lower values. A direct relationship between turbine efficiency and inline, lateral and maximum forces was determined, with more efficient turbines exhibiting increased lateral forces while experiencing minimal changes in inline forces. Maximum forces were found to be more than $40 \%$ higher than the average forces.

This work has also shown that due to the continual increases of computing power available to CFD users, the use of full 3D CFD simulation models for vertical axis turbines is possible without the need for unrealistic computational resources or time requirements. When combined with the ability of the 3D models to capture the effects of geometrical changes on power output the optimisation of turbine design using 3D CFD models is now possible within reasonable timeframes.

The CFD simulation methods developed here will in future work be used to evaluate the performance of helical bladed turbines to determine any differences, and thus possible advantages, between helical and straight bladed designs.

\section{Nomenclature}

$\begin{array}{llll}C_{m} & \text { Moment coefficient } & S & \text { Blade span [m] } \\ C_{m_{\text {Min }}} & \text { Minimum moment coefficient } & T R F & \text { Torque ripple factor } \\ C_{m_{\text {Max }}} & \text { Maximum moment coefficient } & V & \left.\text { Inflow velocity [ms }{ }^{-1}\right] \\ C_{p} & \text { Power coefficient } & y+ & \text { dimensionless distance of } 1^{\text {st }} \text { cell height to wall } \\ F_{I} & \text { Inline force coefficient } & \lambda & \text { Tip speed ratio } \\ F_{L} & \text { Lateral force coefficient } & \rho & \text { Density }\left[\mathrm{kgm}^{-3}\right] \\ F_{\text {Max }} & \text { Maximum force coefficient } & \omega & \text { Rotation rate }\left[\mathrm{rads}^{-1}\right] \\ r & \text { Radius [m] } & & \end{array}$




\section{References}

[1] IPCC. IPCC Special Report on Renewable Energy Sources and Climate Change Mitigation. Prepared by Working Group III of the Intergovernmental Panel on Climate Change [Edenhofer O, Pichs-Madruga R, Sokona Y, Seyboth K, Matschoss P, Kadner S, Zwickel T, Eickemeier P, Hansen G, Schlömer S, von Stechow C(eds)]. Cambridge University Press, Cambridge, United Kingdom and New York, NY, USA. 2011.

[2] CSIRO. Ocean Renewable Energy: 2015:2050. Available from: <www. http://www.csiro.au/Organisation-Structure/Flagships/Energy-Flagship/Ocean-renewableenergy.aspx >. Accessed 13/10/2014.

[3] Dai YM, Lam W. Numerical Study of Straight-Bladed Darrieus-Type Tidal Turbine. Proceedings of the institution of civil engineers. Energy 2009(162):67-76.

[4] Kirke, BK. Tests on ducted and bare helical and straight blade Darrieus hydrokinetic turbines. Renewable Energy 2011;36(11):3013-3022.

[5] Scheurich F, Fletcher TM, Brown R. Simulating the aerodynamic performance and wake dynamics of a vertical-axis wind turbine. Journal of Wind Energy 2010;14:159-177.

[6] New Energy Corporation. Available from: <www. http://www.newenergycorp.ca〉. Accessed $13 / 10 / 2014$.

[7] Paraschivoiu I. Wind Turbine Design: With Emphasis on Darrieus Concept. Quebec, Canada: Polytechnic International Press; 2002.

[8] Marsh P, Ranmuthugala D, Penesis I, Thomas G. Performance predictions of a straight-bladed vertical axis turbine using double-multiple streamtube and computational fluid dynamics. The Journal of Ocean Technology 2013;8(1):87-103.

[9] Templin RJ. Aerodynamic Performance Theory for the NRC Vertical-Axis Wind Turbine (LTRLA-160). Ottawa, Canada: National Research Council of Canada; 1974.

[10] Li Y, Calisal S. Three-dimensional effects and arm effects on modeling a vertical axis tidal current turbine. Renewable Energy 2010;35:2325-2334. 
[11] Li Y, Calisal SM. Numerical Analysis of the characteristics of vertical axis tidal current turbines. Renewable Energy 2010;35:435-442.

[12] Malipeddi AR, Chatterjee D. Influence of duct geometry on the performance of Darrieus hydroturbine. Renewable Energy 2012;43:292-300.

[13] Li C, Zhu S, Xu Y, Xiao Y. 2.5D large eddy simulation of vertical axis wind turbine in consideration of high angle of attack flow. Renewable Energy 2013;51:317-330.

[14] Castelli MR, Ardizzon G, Battisti L, Benini E, Pavesi G. Modeling strategy and numerical validation for a Darrieus vertical axis micro-wind turbine. In: ASME 2010 International Mechanical Engineering Congress and Exposition, 2010. Vancouver, British Columbia, Canada, IMECE201039548.

[15] Marsh P, Ranmuthugala D, Penesis I, Thomas G. Three dimensional numerical simulations of a straight-bladed vertical axis tidal turbine. In: $18^{\text {th }}$ Australasian Fluid Mechanics Conference 2012. Launceston, Tasmania.

[16] Nabavi Y. Numerical study of the Duct Shape Effect on the Performance of a Ducted Vertical Axis Tidal Turbine. MSC dissertation, University of British Columbia; 2008.

[17] Rosetti A, Pavesi G. Comparison of different numerical approaches to the study of the HDarrieus turbines start-up. Renewable Energy 2013;50:7-19.

[18] Nobile R, Vahdati M, Barlow J, Mewburn-Crook A. Dynamic stall for a Vertical Axis Wind Turbine in a two-dimensional study. In: World Renewable Energy Congress 2011 [Sweden].

[19] Ansys Inc. ANSYS CFX-Solver Theory Guide: Release 13.0, 2010. Canonsburg, Pennsylvania.

[20] Rawlings G. Parametric Characterization of an Experimental Vertical Axis Hydro Turbine. MSC dissertation, University of British Columbia; 2008.

[21] Ansys Inc. ANSYS Meshing User’s Guide: Release 13.0, 2010. Canonsburg, Pennsylvania.

[22] Lain S. Simulation and Evaluation of a Straight-Bladed Darrieus-type Cross Flow Marine Turbine. Journal of Scientific and Industrial Research 2010;69:906-912. 
[23] Gretton GI. The hydrodynamic analysis of a vertical axis tidal turbine. PhD dissertation, University of Edinburgh; 2009.

[24] Menter FR. Two-Equation Eddy-Viscosity Turbulence Models for Engineering Applications. AIAA J 1994;32(8):1598-1605.

[25] Sorensen. CFD Modelling of Laminar-turbulent Transition for Airfoils and Rotors Using the $\gamma$ $\operatorname{Re}_{\theta}$ Model. Wind Energy 2009;12:715-733.

[26] Bahaj AS, Molland AF, Chaplin, JR, Batten WMJ. Power and thrust measurements under various hydrodynamic flow conditions in a cavitation tunnel and a towing tank. Renewable Energy 2007;32:407-426.

[27] Leu TS, Yu JM, Hu CC, Miau JJ, Liang SY, Li JY, Cheng JC, Chen SJ. Experimental Study of Free Stream Turbulence Effects on Dynamic Stall of Pitching Airfoil by using Particle Image Velocimetry. Applied Mechanics and Materials 2012;225:103-108.

[28] Maganga F, Germain G, King J, Pinon G, Rivoalen E. Experimental characterisation of flow effects on marine current turbine behaviour and on its wake properties. IET Renewable Power Generation 2010;4(6):498-509.

[29] Maitre T, Amet E, Pellone C. Modeling of the flow in a Darrieus water turbine: Wall grid refinement analysis and comparison with experiments. Renewable Energy 2013;51:497-512.

[30] McLaren KW. A numerical and experimental study of unsteady loading of high solidity vertical axis wind turbines. PhD dissertation, McMaster University; 2011.

[31] Danao, LA. The Influence of Unsteady Wind on the Performance and Aerodynamics of Vertical Axis Wind Turbines. PhD dissertation. University of Sheffield, 2012.

[32] Ferreira, C.J. The near wake of a VWAT: 2D and 3D views of the VWAT aerodynamics. PhD dissertation. Delft University of Technology [2009]

[33] Rawlings GW, Alidadi M, Klaptocz VR, Nabavi Y, Li Y, Mikkelsen J, Calisal SM. Application of End Plates for Vertical Axis Hydro Turbine Performance Enhancement. In: Proceedings of the Eighteenth (2008) International Offshore and Polar Engineering Conference 2008. Vancouver, Canada. 
[34] Mittal S, Kumar B. Flow past a rotating cylinder. Journal of Fluid Mechanics 2003;476:303-334.

[35] Reuter RC, Worstell MH. Torque Ripple in a Vertical Axis Turbine. Sandia Report SAND780577. Albuquerque, New Mexico.

[36] Winchester J, Quayle SD. Torque ripple and power in a variable pitch vertical axis tidal turbine. In: $9^{\text {th }}$ European Wave and Tidal Energy Conference 2011. Southampton, United Kingdom.

[37] Shiono M, Suzuki K, Kiho S. Output Characteristics of Darreius Water Turbine with Helical Blades for Tidal Current Generation. In: Proceedings of the Twelfth (2002) International Offshore and Polar Engineering Conference 2002. Kitakyushu, Japan.

[38] Elmiligui A, Abdol-Hamid KS, Massey SJ, Pao SP. Numerical Study of Flow Past a Circular Cylinder Using RANS, Hybrid RANS/LES and PANS Formulations. AIAA Paper 2004-4959.

[39] Bachant P. Experimental Investigation of Helical Cross-Flow Axis Hydrokinetic Turbines, including Effects of Waves and Turbulence. MSC dissertation, University of New Hampshire; 2011. 